Psychologisches Laboratorium der Universität Utrecht

(Direktor: Prof. Dr F. J. J. BuYTendiJK)

\title{
PHÄNOMENALE GESCHWINDIGKEITSUNTERSCHIEDE BEI BEWEGUNGEN IN VERSCHIEDENEN RICHTUNGEN
}

\author{
voN
}

H. C. VAN DER MEER

\section{Fragestellung}

J. F. Brown beschrieb in seinen Aufsätzen „Über gesehene Geschwindigkeiten" (Ps. Forschung 10, 1927) und „The visual perception of velocity“ (Ps. Forschung 14, 1931) die Faktoren, welche die phänomenale Geschwindigkeit sich bewegender Objekte beeinflussen. In einem dieser Versuche erwähnt er einen Unterschied zwischen ciner horizontalen und einer vertikalen Bewegung, und zwar ist bei gleicher objektiver Geschwindigkeit die vertikale Bewegung phänomenal schneller als die horizontale $\left(V_{h} / V_{v}=1.30\right)$. Die phänomenale Geschwindigkeit einer Bewegung, die unter einem Winkel von $45^{\circ}$ mit der Horizontalen und der Vertikalen verläuft, liegt in der Mitte beider $\left(V_{d} / V_{v}=1.10\right)$.

Dies führt zu der Frage nach dem Einfluss der Orientierung auf die phänomenale Geschwindigkeit. M.a.W. ist eine objektiv vertikale Bewegung in dem Sinne, dass dieser phänomenale Geschwindigkeitsunterschied beibehalten bleibt, immer vertikal unabhängig von der Haltung des Kopfes oder der Stellung des Körpers der Versuchsperson bezüglich der Gravitation?

Die zweite Frage, mit der wir uns beschäftigen wollen, ist: gibt es mehrere Asymmetrien in der phänomenalen Geschwindigkeit der Bewegungen, die in den Hauptrichtungen unseres visuellen Wahrnehmungsraumes verlaufen? D.h. gibt es bei gleicher objektiver Geschwindigkeit phänomenale Geschwindigkeitsunterschiede einerseits zwischen einer nach rechts und einer nach links gerichteten horizontalen Bewegung, anderseits zwischen einer nach oben und einer nach unten gerichteten vertikalen Bewegung?

Die beiden Fragen wollen wir in den nachstehenden Paragraphen behandeln, zuerst aber die befolgte Methode und die Apparatur beschreiben.

\section{Methode}

Die Versuchspersonen erhielten den Auftrag, die Geschwindigkeit von zwei sich bewegenden Objekten miteinander zu vergleichen. Es war dabei nicht gestattet, Hilfsmittel wie z.B. Zählen u.ä. zu benutzen, sondern die Versuchspersonen sollten nur den unmittelbaren Eindruck, den die Schnelligkeit auf sie machte, beobachten. 
Die sich bewegenden Objekte waren zwei Lichtpunkte mit einem Durchmesser von $14 \mathrm{~mm}$, die auf einen Schirm in einer frontal-parallelen Fläche nacheinander projiziert wurden (nur in den ersten orientierenden Versuchen war der Durchmesser grösser). Dieser Schirm war aus einem Bogen schwarzer Pappe von $55 \times 65 \mathrm{~cm}$ hergestellt, in dessen Mitte ein Viereck von $15 \mathrm{~cm}^{2}$ ausgeschnitten war, auf das Schreibmaschinenpapier geklebt wurde, so dass die Lichtpunkte auf der Hinterseite deutlich wahrnehmbar waren. Die Versuchspersonen sassen hinter dem Schirm in einer Entfernung von $1.20 \mathrm{~m}$. Der Schirmausschnitt von $15 \times 15 \mathrm{~cm}$, auf dem sich die Lichtpunkte bewegten, befand sich ungefähr in Augenhöhe. Die Bewegungen der Lichtpunkte kreuzten sich in der Mitte des Feldes, so dass der Schnittpunkt der horizontalen und der vertikalen Bewegung mit demjenigen der Diagonalen des Schirmausschnittes zusammenfiel.

Die Lichtpunktquelle war eine Glühbirne von $0.45 \mathrm{~A}, 8 \mathrm{~V}$, die sich in einer kleinen Hülse befand und vor welcher eine positive Linse angebracht war. Das austretende Strahlenbündel fiel auf einen Spiegel, vor dessen unmittelbarer Nähe noch eine zweite positive Linse aufgestellt war. Dieser Spiegel war an einer Scheibe befestigt, welche durch einen Elektromotor in Bewegung gesetzt werden konnte, dessen Geschwindigkeit variabel war. Das Lichtbündel der Glühbirne wurde von dem rotierenden Spiegel als ein sich bewegender Lichtpunkt auf den Schirm projiziert. Der Abstand vom Schirm bis zu den Spiegeln betrug ca. $1.65 \mathrm{~m}$.

Die Grösse der Lichtpunkte konnte durch ein Diaphragma vor der ersten Linse beliebig geändert werden; die Lichtintensität der beiden Punkte war durch Einschaltung eines Widerstandes zu regeln. Die Abstände von der ersten Linse bis zur Glühbirne und von der zweiten Linse bis zum Spiegel, sowie der Abstand der Glühbirne bis zum Spiegel und die Stärke der Linsen sollten so bestimmt werden, dass die Lichtpunkte auf dem Schirm völlig scharf und identisch waren.

Die Geschwindigkeit der horizontalen Bewegung wurde konstant gehalten, die der vertikalen Bewegung war variabel. Die Bewegungen wurden sofort nacheinander gezeigt.

Die Versuche wurden im Dunkeln vorgenommen. Da aber die Lichtbündel der beiden Glühbirnen ein sehr schwaches Licht verbreiteten, war es nicht vollkommen finster, sondern konnten die Versuchspersonen - nachdem sie sich an die Dunkelheit gewöhnt hatten - die Umrisse der Objekte im Zimmer sehr verschwommen wahrnehmen. Wahrscheinlich entspricht dies ungefähr den Bedingungen Browns, der seine Versuche auch nicht in völliger Finsternis angestellt hat.

Die Versuche wurden in solcher Weise vorgenommen, dass die Versuchsperson, die zwei nacheinander erscheinenden Geschwindigkeiten miteinander zu vergleichen und solange der Vl. Anweisungen für Veränderung der zweiten Geschwindigkeit zu geben hatte, bis ihr beide gleich erschienen. Für jede Vp. wurden 6 bis 8 Einstellungen gemacht, deren Durchschnittswerte in den nachstehenden Tabellen angeführt sind. Es wurde dabei abwechselnd angefangen mit einer Geschwindigkeit, die entweder bedeutend grösser oder bedeutend kleiner war als die Standardgeschwindigkeit. Dadurch hatte die Vp. zunächst den Eindruck eines deutlichen Geschwindigkeitsunterschiedes, worauf dann dic Anzäherung an Glcichhcit erfolgen konnte. Wenn die Vp. meinte, dass die Geschwindigkeiten der beiden Punkte übereinstimmten, wurde die Geschwindigkeit der Punkte festgestellt, indem die Zeit, die der Punkt horizontal über einen Abstand von $80 \mathrm{~cm}$, vertikal über einen Abstand von $60 \mathrm{~cm}$ durchlief, mit einer Stoppuhr gemessen wurde. 
Obgleich die Versuche im Dunkeln erfolgten, und die Vl. also die Geschivindigkeit auf der Skalaeinteilung des Motors nicht ablesen konrte, wurde bei jeder $V_{p}$. kontrolliert, ob von der Vl. eine suggestive Wirkung ausgehen könne. Zu diesem Zwecke veränderte die Vl., wenn der Geschwindigkeitsunterschied für die Vp. nur noch schr gering war, einige Male die Geschwindigkeit der Variabelen, gegensätzlich der Anweisung der Vp. In keinem einzigen Falle konnte die Vl. auf diese Weise das Urteil der Vp. beeinflussen.

Da bei diesen Untersuchungen vorausgesetzt wird, dass man dazu fähig ist, zwei gesehene Geschwindigkeiten einigermassen genau miteinander zu vergleichen, wurdc dies zucrst kontrolliert. Bci zwei Vpn. wurden auf obenerwähnte Weise 6 Einstellungen gemacht um die subjektive Geschwindigkeitsgleichheit zweier sich in gleicher Richtung bewegender Objekte zu bestimmen. Es stellte sich heraus, dass der Sukzessivvergleich zweier Geschwindigkeiten in gleicher Richtung ganz gut gelingt; für beide Vpn. wich der Unterschied der objektiven Geschwindigkeit des zweiten Punktes bei subjektiver Gleichheit weniger als $1 \%$ von der Standardgeschwindigkeit ab.

Die Vpn. waren zum Teil Studenten, die am Anfang ihres Studiums standen und zum Teil Angestellte des Laboratoriums. Gerne sage ich ihnen meinen Dank für ihre Bereitheit, sich als Vp. zur Verfügung zu stellen.

\section{Versuche ÜBer horizontale UND vertikale BEWEgung}

Brown, der in seinen Versuchen über die phänomenale Geschwindigkeit sich bewegender Objekte mit schwarzen Punkten arbeitete, die sich auf einem beleuchteten Feld bewogen, stellte fest, dass eine Bewegung in vertikaler Richtung phänomenal schneller ist als eine Bewegung in horizontaler Richtung $\left(V_{h} / V_{v}=1.30\right)$. Die bewegenden Objekte in meinen Versuchen waren dagegen Lichtpunkte, die auf ein weisses Feld projiziert wurden. Zuerst musste also untersucht werden, ob der von Brown entdeckte Unterschied in phänomenaler Geschwindigkeit zwischen einer horizontalen und einer vertikalen Bewegung auch unter diesen Bedingungen auftrat.

$\mathrm{Zu}$ diesem $\mathrm{Zwecke}$ wurden zunächst einige orientierende Versuche angestellt.

Versuch 1. Lichtpunkte mit einem Durchmesser von $40 \mathrm{~mm}$ wurden auf einen weissen Schirm von $46.2 \times 46.2 \mathrm{~cm}$ projiziert. Der Beobachtungsabstand war $1.20 \mathrm{~m}$. Die Geschwindigkeit der Standardbewegung, von rechts nach links laufend, $V_{h}$, war $5.5 \mathrm{~cm} / \mathrm{sec}$. Die vertikale Bewegung lief von unten nach oben. Die Wahrnehmung war binokular, ohne Fixation.

'TABELLL' 1

Feldgrösse $46.2 \times 46.2 \mathrm{~cm}, V_{h}: 5.5 \mathrm{~cm} / \mathrm{sec}$.

\begin{tabular}{|c|c|c|c|c|c|}
\hline \multicolumn{3}{|c|}{ Beobachtung binokular } & \multicolumn{3}{|c|}{ Beobachtung monokular } \\
\hline Vpn. & $V_{h} / V_{v}$ & $\sigma$ & $\mathrm{V}_{\mathrm{p} n}$. & $V_{h} / V_{v}$ & $\sigma$ \\
\hline $\begin{array}{l}\text { vdM } \\
S\end{array}$ & $\begin{array}{l}1.30 \\
1.12\end{array}$ & $\begin{array}{l}1.02 \\
1.56\end{array}$ & $\begin{array}{l}\mathrm{vdM} \\
\mathrm{S}\end{array}$ & $\begin{array}{l}1.24 \\
1.11\end{array}$ & $\begin{array}{l}0.81 \\
0.97\end{array}$ \\
\hline
\end{tabular}


Versuch 2. Wie Versuch 1; die Wahrnehmung jedoch monokular, ohne Fixation.

$V_{h} / V_{v}$ bezeichnet das Verhältnis der objektiven Geschwindigkeiten bei subjektiver Gleichheit. Die Zeitmessungen bilden die Basis für die Berechnung der Streuungen.

Versuch 3. $V_{h}=21 \mathrm{~cm} / \mathrm{sec}$; im übrigen wie Versuch 1.

Versuch 4. $V_{h}=21 \mathrm{~cm} / \mathrm{sec}$; im übrigen wie Versuch 2.

TABELLE 2

Feldgrösse $46.2 \times 46.2 \mathrm{~cm}, V_{h}: 21 \mathrm{~cm} / \mathrm{sec}$.

\begin{tabular}{c|c|c||c|c|c}
\multicolumn{2}{c||}{ Beobachtung binokular } & \multicolumn{3}{c}{ Beobachtung monokular } \\
\hline Vpn. & $V_{h} / V_{v}$ & $\sigma$ & Vpn. & $V_{h} / V_{v}$ & $\sigma$ \\
\hline vdM & 0.80 & 0.15 & vdM & 0.81 & 0.18 \\
S & 0.90 & 0.73 & $\mathrm{~S}$ & 0.80 & 0.20
\end{tabular}

Vergleichen wir die Resultate aus den Tabellen 1 und 2, so ergibt sich daraus, dass bei einer Geschwindigkeit der Standardbewegung von 5.5 $\mathrm{cm} / \mathrm{sec}$, die phänomenale Geschwindigkeit der vertikalen Bewegung die grössere ist; jedoch bei einer Geschwindigkeit von $21 \mathrm{~cm} / \mathrm{sec}$. diejenige der horizontalen Bewegung.

Es wurden nun einige Anderungen in der Versuchsanordnung vorgenommen um zu prüfen, ob dieses Resultat persistent sei. Der Schirm bestand jetzt aus schwarzer Pappe von $55 \times 65 \mathrm{~cm}$, aus dessen Mitte ein Viereck von $30 \mathrm{~cm}^{2}$ ausgeschnitten wurde, das mit weissem Schreibmaschinepapier beklebt wurde.

Versuch 5. Feldgrösse $30 \times 30 \mathrm{~cm}$, Punktdurchmesser $30 \mathrm{~mm}$, Beobachtungsabstand $1.20 \mathrm{~m}$. Wahrnehmung binokular, ohne Fixation. Die Standardbewegung lief von rechts nach links, die Variabele von unten nach oben. $V_{h}: 5 \mathrm{~cm} / \mathrm{sec}$.

Versuch 6. Wie Versuch $5 ; V_{h}: 10 \mathrm{~cm} / \mathrm{sec}$.

Versuch 7. Wie Versuch $5 ; V_{h}: 20 \mathrm{~cm} / \mathrm{sec}$.

Die Ergebnisse dieser drei Versuche sind in Tabelle 3 zusammengefasst. In diesen und den nachfolgenden Versuchen wurde die Zeit für die horizontale Bewegung über einen Abstand von $80 \mathrm{~cm}$, für die vertikale Bewegung über $60 \mathrm{~cm}$ gemessen. Die Zeitmessungen bilden die Basis für die Berechnung der Streuungen. 
TABELLE 3

Feldgrösse $30 \times 30 \mathrm{~cm}$, Beobachtung binokular, Bewegungsrichtungen $\leftarrow \uparrow$

\begin{tabular}{l|l|l||l|l|l||l|l|l}
\hline \multicolumn{3}{c|}{$V_{h}: 5 \mathrm{~cm} / \mathrm{sec}}$. & \multicolumn{3}{c||}{$V_{h}: 10 \mathrm{~cm} / \mathrm{sec}}$. & \multicolumn{3}{c}{$V_{h}: 20 \mathrm{~cm} / \mathrm{sec}}$. \\
\hline Vpn. & $V_{h} / V_{v}$ & $\sigma$ & Vpn. & $V_{h} / V_{v}$ & $\sigma$ & Vpn. & $V_{h} / V_{v}$ & \multicolumn{1}{c}{$\sigma$} \\
\hline vdM & 1.10 & 0.24 & vdM & 0.98 & 0.28 & vdM & 0.90 & 0.09 \\
S & 1.12 & 1.56 & $\mathrm{~S}$ & 0.90 & 0.45 & $\mathrm{~S}$ & 0.95 & 0.21 \\
Y & 1.13 & 1.50 & $\mathrm{Bl}$ & 1.10 & 1.02 & $\mathrm{vG}$ & 0.90 & 0.37 \\
\hline $\mathrm{W}$ & 0.87 & 0.14 & $\mathrm{~W}$ & 0.90 & 0.14 & $\mathrm{~W}$ & 1.23 & 0.38 \\
$\mathrm{C}$ & 1.06 & 1.66 & & & & $\mathrm{C}$ & 1.50 & 0.25 \\
$\mathrm{~F}$ & 0.75 & 1.60 & & & & $\mathrm{Ba}$ & 1.11 & 0.32 \\
$\mathrm{~B}$ & 0.97 & 0.37 & & & & & & \\
$\mathrm{Z}$ & 0.90 & 0.62 & & & & & &
\end{tabular}

Die Ergebnisse in der Tabelle 3 entsprechen jedoch dem Resultat Browns nicht. Der phänomenale Geschwindigkeitsunterschied zwischen der horizontalen und der vertikalen Bewegung ist weniger ausgeprägt. Die Geschwindigkeit, bzw. die Expositionszeit, scheint die Richtung dieses Unterschiedes zu beinflussen, und ausserdem sind die interindividuellen Unterschiede gross. Muss man nun etwa zwei Typen Vpn. annehmen, einen der bei geringerer Geschwindigkeit die horizontale Bewegung, bei grösserer Geschwindigkeit die vertikale Bewegung überschätzt, während bei dem anderen Typus diese Illusionen gerade umgekehrt sind? Für diese Annahme könnte sprechen, dass nach Sleight und Austin (J. of Psychology 1952) die klassische, statische horizontal-vertikale Illusion in der Reihe ViereckRechteck nicht allgemein gültig wäre, doch dass es Personen mit einer klassischen und einer kontraklassischen Illusion, sowie auch solche ohne Illusion, gäbe. Es ist nicht ohne weiteres klar, wie diese Tendenz auf die hier beschriebene Bewegungsillusion von Einfluss sein könnte und warum der Geschwindigkeitsfactor, bzw. die Expositionszeit, eine umkehrende Wirkung auf das Verhältnis der Illusionen ausüben würde.

$\mathrm{Da}$ es in erster Linie die Absicht war, zu prüfen, ob unter den hier geltenden Bedingungen eine gleiche Bewegungsillusion als von Brown entdeckt wurde, vorkomme, wollen wir jetzt dieses Problem nicht weiter verfolgen, sondern versuchen, ob wir, indem wir die Momente Geschwindigkeit, Grösse des bewegenden Punktes und Feldgrösse möglichst genau mit den von Brown verwendeten Grössen in Ubereinstimmung bringen, eine gleich grosse Illusion ermitteln können, die annähernd für alle Vpn. gültig ist.

Versuch 8. Feldgrösse $15 \times 15 \mathrm{~cm}$, Punktdurchmesser $14 \mathrm{~mm}$, 
Beobachtungsabstand $1.20 \mathrm{~m} . V_{h}: 10 \mathrm{~cm} / \mathrm{sec}$. Die Wahrnehmung war binokular, ohne Fixation.

Die Pfeile in den Tabellen bezeichnen die Richtungen der Bewegungen.

TABELLE 4

Feldgrösse $15 \times 15 \mathrm{~cm}$, binokulare Beobachtung. $V_{h}: 10 \mathrm{~cm} / \mathrm{sec}$. Bewegungsrichtungen $\leftarrow \uparrow$

\begin{tabular}{l|c|c}
\hline \hline Vpn. & $V_{h} / V_{v}$ & $\sigma$ \\
\hline vdM & 1.03 & 0.25 \\
$W$ & 1.02 & 0.14 \\
dJ & 1.14 & 0.39 \\
$V$ & 1.03 & 0.44
\end{tabular}

Versuch 9. Wie Versuch 8, mit dem Unterschied, dass die horizontale Bewegung von links nach rechts verlief.

Versuch 10. Wie Versuch 9, jedoch die vertikale Bewegung von oben nach unten verlaufend.

TABELLE 5

Felclgrösse $15 \times 15 \mathrm{~cm}, V_{h}: 10 \mathrm{~cm} / \mathrm{sec}$. Beobaclitung binokular

\begin{tabular}{c|c|c}
\hline \hline Bewcoungsrichtungen & $\rightarrow \uparrow$ & $\rightarrow \downarrow$ \\
\hline Vpn. & $V_{h} / V_{n}$ & $V_{h} / V_{v}$ \\
\hline vdM & 1.31 & 1.29 \\
W & 1.24 & 1.24 \\
d.J & 1.34 & 1.35 \\
$V$ & 1.36 & 1.36
\end{tabular}

Aus Tabelle 5 ersehen wir, dass die von Brown entdeckte Bewegungsillusion unter diesen Bedingungen tatsächlich auftritt.

Aus den Versuchen 8 bis 10 ergibt sich demnach, dass man unter den hier beschriebenen Bedingungen nicht im allgemeinen von einer horizontal-vertikalen Bewegungsillusion reden kann, sondern dass diese Illusion nur zwischen einer nach rechts gerichteten horizontalen und einer vertikalen Bewegung auftritt. Zwischen einer nach oben und einer nach unten gerichteten Bewegung scheint kein Unterschied zu bestehen.

Im nächsten Paragraphen werde ich auf den hier gefundenen Unterschied zwischen einer nach rechts und einer nach links gerichteten horizontalen Bewegung zurückkommen; dabei werde ich dann zugleich den Einfluss des Zeitfehlers, der in diesen Versuchen nicht berücksichtigt 
wurde, in Betracht ziehen. Jetzt wollen wir die weiteren Versuche zwischen der horizontalen und der vertikalen Bewegung verfolgen.

Versuch 11. Wie die Versuche 8 und 9; die Wahrnehmung jedoch monokular, ohne Fixation.

TABELLE 6

Feldgrösse $15 \times 15 \mathrm{~cm}, V_{h}: 10 \mathrm{~cm} / \mathrm{sec}$. Beobachtung monokular

\begin{tabular}{c|c|c}
\hline \hline Bewegungsrichtungen & $\rightarrow \uparrow$ & $\leftarrow \uparrow$ \\
\hline Vpn. & $V_{h} / V_{v}$ & $V_{h} / V_{\diamond}$ \\
\hline vdM & 1.38 & 1.08 \\
S & 1.23 & 1.11 \\
dV & 1.33 & 1.17 \\
vG & 1.38 & 1.11 \\
R & 1.34 & 1.06 \\
Sch & 1.32 & 1.17
\end{tabular}

Vergleichen wir die Tabellen 4 und 5 mit 6, so ersehen wir, dass zwischen der Grösse der Täuschung bei monokularer und binokularer Beobachtung kein wesentlicher Unterschied besteht.

Versuch 12. Wie die Versuche 8 und 9, jetzt aber mit Fixation.

Als Fixationspunkt wählte ich einen leuchtenden Punkt mit einem Durchmesser von $4 \mathrm{~mm}$, der in der Mitte des Feldes, also im Schnittpunkt der Bahnen, welche die beiden Punkte durchliefen, angebracht wurde.

TABELLE 7

Feldgrösse $15 \times 15 \mathrm{~cm}, V_{h}: 10 \mathrm{~cm} / \mathrm{sec}$. Beobachtung mit Fixationspunkt

\begin{tabular}{c|c|c}
\hline \hline Bewegungsrichtungen & $\rightarrow \uparrow$ & $\leftarrow \uparrow$ \\
\hline Vpn. & $V_{h} / V_{v}$ & $V_{h} / V_{v}$ \\
\hline vdM & 1.18 & 1.17 \\
$\mathrm{~S}$ & 1.17 & 1.16 \\
$\mathrm{Li}$ & 1.26 & 1.25 \\
$\mathrm{M}$ & 1.30 & 1.25
\end{tabular}

Aus Tabelle 7 geht hervor, dass bei Fixation der Mitte der beiden Bewegungsbahnen der phänomenale Geschwindigkeitsunterschied zwischen der horizontalen und der vertikalen Bewegung herabgesetzt ist. Der phänomenale Geschwindigkeitsunterschied zwischen einer nach rechts und einer nach links gerichteten Bewegung ist jetzt aufgehoben.

Jetzt wollen wir untersuchen, wie es sich mit dem phänomenalen Ge- 
schwindigkeitsunterschied zwischen einer horizontalen und einer vertikalen Bewegung verhält, wenn die Lage des Körpers oder die Haltung des Kopfes nicht mehr vertikal ist. Dazu wurden die nachfolgenden Versuche angeordnet.

Versuch 13. Die Vp. lag auf der linken Seite auf einem Sofa und zwar so, dass die Verbindungslinie zwischen den Augen vertikal war. Die Mitte des Schirms war in Augenhöhe. Die übrigen Verhältnisse waren wie in den Versuchen 8 bis 10 . Die Ergebnisse sind in Tabelle 8 zusammengestellt.

TABELLE 8

In liegender Haltung. $V_{h}: 10 \mathrm{~cm} / \mathrm{sec}$.

\begin{tabular}{c|c|c|c}
\hline \hline Bewegungsrichtungen & $\rightarrow \uparrow$ & $\rightarrow \downarrow$ & $\leftarrow \uparrow$ \\
\hline Vpn. & $V_{h} / V_{v}$ & $V_{h} / V_{v}$ & $V_{h} / V_{v}$ \\
\hline vdMl & 0.81 & 0.81 & 0.85 \\
$W$ & 1.11 & 1.00 & 1.00 \\
Schl & 0.97 & 0.89 & 0.96
\end{tabular}

Aus den Resultaten von Tabelle 8, und mehr noch aus den einzelnen Einstellungen der Vpn., geht hervor, dass man bei horizontaler Körperlage im Dunkeln seine normale Orientierung zum Teil verliert. Die Orientierung ist laut der Grösse der Geschwindigkeiten, die von den Vpn. als gleich beurteilt wurden, äusserst labil; bald scheint die normale, bald die Orientierung gemäss der liegenden Haltung, wobei also die objektive Horizontale die subjektive Vertikale wird, zu überwiegen. Es besteht eine grosse Unsicherheit über das Urteil, die in keinem der vorherigen Versuche vorkam. Dieselbe Geschwindigkeit wurde das eine Mal als viel zu langsam, sofort danach als viel zu schnell bezüglich der Standardgeschwindigkeit beurteilt.

Die Labilität der Orientierung geht auch aus dem Folgenden hervor. Die erste Vp. aus Tabelle 8 versuchte die Orientierung, wobei die objektive Horizontale als Vertikale aufgefasst wurde, beizubehalten. Dies gelang insofern, dass die Geschwindigkeit der Variabelen in den verschiedenen Einstellungen sehr konstant war. Die Streuungen waren nämlich bzw. 0; 0.08 und 0 . Es erfolgte aber ein leichtes Schwindelgefühl. Die Illusion war in diesem Falle der Illusion bei vertikaler Körperlage entgegengesetzt. Wenn man beachtet, dass der Zeitfehler auf die Illusionen in den beiden Situationen, d.h. in aufrechter und in liegender Haltung, einen entgegengesetzten Einfluss ausübt, so scheint die Annahme, dass die Grössen der 
Illusionen in den beiden Körperlagen sich ziemlich genau entsprechen, berechtigt.

Hieraus geht also hervor, dass dasjenige was als horizontal oder vertikal aufgefasst wird, nicht von dem Augenstand abhängig ist, sondern durch die Bedeutung, die man den Hauptdimensionen des Raumes verleiht, bedingt wird. Bei unvoreingenommenen Vpn. überwiegt bald die eine, bald die andere Orientierung.

Aus Versuch 13 geht hervor, dass im Dunkeln in liegender Haltung eine gewisse Labilität der Orientierung besteht, wobei die Orientierung, bei der die objektive Vertikale als horizontal gesehen wird, vorzuherrschen scheint. Es fragt sich nun, wie sich dies im Hellen verhalten wird.

Versuch 14. Die Vpn. sollten die Geschwindigkeiten sowohl im Dunkeln wie auch unter einer schwachen Beleuchtung miteinander vergleichen. Die Beleuchtung war nicht so stark, dass man darin hätte lesen können; die Umrisse des Zimmers und die Gegenstände waren jedoch ganz deutlich wahrnehmbar. Die Vpn. lagen wie in Versuch 13 auf dem Sofa.

TABELLE 9

Vergleich zwischen Beobachtung im Dunkeln und im Hellen

\begin{tabular}{c|c|c}
\hline & im Dunkeln & im Hellen \\
\hline Bewegungsrichtungen & $\rightarrow \uparrow$ & $\rightarrow \uparrow$ \\
\hline Vpn. & $V_{h} / V_{v}$ & $V_{h} / V_{v}$ \\
\hline vdM & 0.81 & 1.03 \\
V & 0.92 & 1.17 \\
S & 0.90 & 1.03
\end{tabular}

Vergleich der Quotienten $V_{h} / V_{v}$ im Dunkeln und bei schwacher Beleuchtung ergibt also (Tab. 9), dass in liegender Haltung die normale Horizontal-Vertikalorientierung im Hellen besser beibehalten bleibt als im Dunkeln, jedoch auch nicht vollkommen. Was als horizontal oder vertikal aufgefasst wird, ist also nicht vom Augenstand abhängig, sondern wird durch die Struktur des ganzen Gesichtsfeldes mitbedingt.

Versuch 15. Es wurde untersucht, welcher der Einfluss auf den phänomenalen Geschwindigkeitsunterschied einer horizontalen und einer vertikalen Bewegung ist, wenn man diese unter einem Kopfstand von $45^{\circ}$ seitwärts, und zwar entweder mit dem Kinn nach rechts oder nach links gerichtet, beobachtet.

Die Wahrnehmung war binokular, ohne Fixation. 
In Tabelle 10 sind die Resultate zusammengestellt. Zum Vergleich wurden auch die Werte des Verhältnisses $V_{h} / V_{v}$ bei normalem Kopfstand bestimmt.

TABELLE 10

Vergleich bei aufrechter und geneigter Kopfhaltung. Beobachtung binokular

\begin{tabular}{c|c|c|c|c}
\hline \hline Kopf haltung & \multicolumn{2}{|c|}{ aufrecht } & $45^{\circ}$ n.l. & $45^{\circ}$ n.r. \\
\hline Bewegungsrichtungen & $\rightarrow \uparrow$ & $\leftarrow \uparrow$ & $\rightarrow \uparrow$ & $\rightarrow \uparrow$ \\
\hline Vpn. & $V_{h} / V_{v}$ & $V_{h} / V_{v}$ & $V_{h} / V_{v}$ & $V_{h} / V_{v}$ \\
\hline vdM & 1.38 & 1.06 & 1.06 & 1.09 \\
W & 1.24 & 1.02 & 1.14 & 1.13 \\
vBr & 1.38 & 1.09 & 1.10 & 1.09
\end{tabular}

Versuch 16. Wie Versuch 15, die Wahrnehmung monokular (rechtes Auge).

TABELLE 11

Vergleich bei aufrechter und geneigter Kopfhaltung. Beobachtung monokular

\begin{tabular}{c|c|c|c|c}
\hline \hline Kopf haltung & \multicolumn{2}{|c|}{ aufrecht } & $45^{\circ}$ n.l. & $45^{\circ}$ n.r. \\
\hline Bewegungsrichtungen & $\rightarrow \uparrow$ & $\leftarrow \uparrow$ & $\rightarrow \uparrow$ & $\rightarrow \uparrow$ \\
\hline Vpn. & $V_{h} / V_{v}$ & $V_{h} / V_{\vartheta}$ & $V_{h} / V_{v}$ & $V_{h} / V_{v}$ \\
\hline vdM & 1.38 & 1.08 & 1.15 & 1.12 \\
$\mathrm{dV}$ & 1.33 & 1.17 & 1.13 & 1.14 \\
Sch & 1.32 & 1.17 & 1.06 & 1.08
\end{tabular}

Der Vergleich der Tabellen 10 und 11 ergibt an erster Stelle, dass es keinen Unterschied macht, ob der Kopf $45^{\circ}$ nach links oder nach rechts gerichtet ist; auch zwischen monokularer und binokularer Beobachtung scheint kein wesentlicher Unterschied zu bestehen.

Es gibt einen geringen Unterschied in phänomenaler Geschwindigkeit zwischen der horizontalen und der vertikalen Bewegung; ein kleiner Unterschied ist aber schon auf Grund des Zeitfehlers zu erwarten.

Im allgemeinen neigten die Vpn. dazu, die beiden Bewegungen symmetrisch in Bezug auf den Kopf bewegen zu sehen, so dass die beiden Bewegungen nicht mehr als horizontal oder vertikal aufgefasst wurden. Man könnte hier also von einer egozentrischen Orientierung sprechen.

Versuch 17. Die sich bewegenden Punkte wurden auf die Decke projiziert. Uber die rotierenden Spiegel wurde ein Schirm aus schwarzer Pappe 
hingestellt, aus dem zwei Schlitze geschnitten wurden, so dass die beiden Bewegungsbahnen an der Decke einander in der Mitte kreuzten. Die Länge der beiden Bahnen an der Decke betrug $18 \mathrm{~cm}$. Der Abstand der Decke bis zu den Spiegeln betrug $1.90 \mathrm{~m}$. Die Geschwindigkeit der Bewegungen wurde nach der Zeitdauer der Bewegung über einen Abstand von $53 \mathrm{~cm}$ berechnet.

Die Vpn. lagen auf einem Sofa; die Bewegungen verliefen etwas hinter den Vpn.

In Tabelle 12 sind die Resultate erwähnt; $V_{v}$ bedeutet die Geschwindigkeit der Bewegung parallel mit der Längenachse des Körpers der Vpn.

TABELLE 12

Bewegungen an der Decke. Beobachtung in liegender Haltungr

\begin{tabular}{c|c|c}
\hline \hline Bewegungsrichtungen & $\rightarrow \uparrow$ & $\rightarrow \downarrow$ \\
\hline Vpn. & $V_{h} / V_{v}$ & $V_{h} / V_{v}$ \\
\hline vdM & 1.38 & 1.37 \\
D & 1.56 & 1.33 \\
vdK & 1.31 & 1.10
\end{tabular}

Die phänomenale Geschwindigkeit der vertikalen Bewegung ist also (Tab. 12) beträchtlich grösser als die der horizontalen Bewegung. Bei den beiden letzten Vpn. aus Tabelle 12 scheint jetzt auch ein phänomenaler Geschwindigkeitsunterschied zwischen der steigenden (sich entfernenden) und der fallenden (sich nähernden) Bewegung vorzuliegen, und zwar wird die steigende Bewegung schneller als die fallende Bewegung gesehen. Im nächsten Abschnitt werden wir dies einer näheren Prüfung unterziehen.

Versuch 18. Die Versuchpersonen sassen jetzt aufrecht und beobachteten die Bewegungen, die schräg über und vor ihnen an der Decke erschienen.

TABELLE 13

Bewegungen an der Decke. Beobachtung aufrecht sitzend

\begin{tabular}{c|c|c}
\hline Bewegungsrichtungen & $\rightarrow \uparrow$ & $\rightarrow \downarrow$ \\
\hline Vpn. & $V_{h} / V_{v}$ & $V_{h} / V_{v}$ \\
\hline \multirow{2}{*}{$\mathrm{Li}$} & 1.04 & 1.00 \\
& 0.91 & 0.96 \\
$\mathrm{vdM}$ & 1.00 & 1.03 \\
$\mathrm{~S}$ & 0.78 & 0.90 \\
& 0.76 & 0.85 \\
Gl & 0.84 & 0.96 \\
& 0.79 & 0.90 \\
& 1.50 & 1.31
\end{tabular}


In Tabelle 13 sind die Durchschnittswerte aus je 8 Einstellungen angeführt. Da die interindividuellen Ergebnisse nicht einstimmig waren und die Vpn. den Auftrag in diesem Falle als sehr schwierig empfanden, weil die Situation so labil war, wurden die Versuche einige Wochen später wiederholt. Dies machte aber keinen grossen Unterschied.

Als besondere Schwierigkeit dieses Versuches galt, dass man die Bewegungen bald an einer frontalparallelen Fläche, wobei die sich nähernde Bewegung als nach oben, die sich entfernende Bewegung als nach unten gerichtet gesehen wurde, bald an der Decke sah. Dies sind selbstverständlich die beiden Äussersten, zwischen denen die Lokalisation erfolgte. Sah man die Bewegungen an der Decke, so wurden sie eher als sagittal wie als vertikal gesehen. Sah man die Bewegungen an einer frontalparallelen Fläche oder zwischen diesen beiden Stellungen, so wurde die objektiv sich nähernde Bewegung als nach oben, die sich entfernende als nach unten gerichtet gesehen. Die Desorientierung war manchmal so gross, dass die Vp. meinte, dass die Richtung der Bewegung während des Versuches nicht konstant bliebe, sondern bald nach oben, bald nach unten verliefe. Die Vp. S meinte - als nach Beendigung des Versuches die sich entfernende und die sich nähernde Bewegung nacheinander gezeigt wurden — dass beide nach oben gerichtet seien, die sich nähernde Bewegung nur etwas ausgeprägter.

Als Folge dieser Labilität und der daraus resultierenden Desorientierung traten weitere störende Phänomene auf. So wurde z.B. die Bewegung von links nach rechts nicht in konstanter Geschwindigkeit gesehen, obgleich dies wohl der Fall war. Bald wurde die Länge der horizontalen Bahn als länger, bald als kürzer als die vertikale, bzw. sagittale beurteilt. Weiter waren die Konstanzphänomenen bedeutend beeinträchtigt.

\section{Versuche Über BeWEgUngen IN ENTGEgengeSETZTEN RichtungeN}

Die im vorigen Paragraphen erwähnten Versuche berechtigten uns einigermassen zur Annahme, dass es einen Unterschied in phänomenaler Geschwindigkeit zwischen einer nach rechts und einer nach links gerichteten Bewegung gäbe. Dagegen wurde kein Unterschied zwischen einer aszendenten und einer deszendenten Bewegung gefunden. Um hierüber mehr Sicherheit zu bekommen, wollen wir in diesem Abschnitt die Bewegungen in entgegengesetzter Richtung auf direktem Wege miteinander vergleichen. An jedem dieser Versuche wurde von $10 \mathrm{Vpn}$. teilgenommen; von jeder Vp. wurden 6 Einstellungen erhalten.

Wir wollen hier auch den Einfluss des Zeitfehlers untersuchen, was in den vorigen Versuchen unterlassen wurde, zum Teil aus praktischen Gründen (zu lange Versuchszeit pro Vp.), zum Teil weil die gefundenen 
Unterschiede derart gross waren, dass diese nicht nur als eine Fơlge des Zeitfehlers betrachtet werden konnten.

Aus technischen Gründen war der Zeitraum, in dem die beiden Bewegungen einander folgten, in diesen Versuchen etwas grösser als in den Experimenten über horizontale und vertikale Bewegung. Daher wird der hier gefundene Zeitfehler wahrscheinlich etwas grösser sein als derjenige, den man in den vorigen Versuchen in Abzug bringen müsste.

Versuch 19a. Feldgrösse $15 \times 15 \mathrm{~cm}$, Punktdurchmesser $14 \mathrm{~mm}$, Beobachtungsabstand $1.20 \mathrm{~m}$. Geschwindigkeit der Standardbewegung $V_{s}: 10 \mathrm{~cm} / \mathrm{sec}$. Die Wahrnehmung war binokular, ohne Fixation.

Die Standardbewegung war von rechts nach links, die Variabele von links nach rechts.

Versuch $19 \mathrm{~b}$. Der Standard von links nach rechts, die Variabele von rechts nach links.

TABELLE 14

Vergleich von zwei horizontalen Bewegungen in entgegengesezter Richtung. $V_{8}: 10 \mathrm{~cm} / \mathrm{sec}$.

\begin{tabular}{c|c|c|c}
\hline Standardrichtung & nach 1. & nach $\mathrm{r}$. & $\begin{array}{c}V_{r} / V_{l} \\
\text { durchschn. }\end{array}$ \\
\hline Vpn. & $V_{r} / V_{s}$ & $V_{s} / V_{l}$ & \\
\hline vdM & 0.99 & 1.07 & 1.03 \\
$\mathrm{Li}$ & 0.97 & 1.08 & 1.02 \\
$\mathrm{~S}$ & 1.03 & 1.11 & 1.07 \\
$\mathrm{Kl}$ & 0.99 & 1.07 & 1.03 \\
$\mathrm{dJ}$ & 0.91 & 1.12 & 1.01 \\
$\mathrm{Wr}$ & 0.96 & 1.12 & 1.03 \\
$\mathrm{Br}$ & 0.93 & 1.02 & 0.98 \\
$\mathrm{R}$ & 0.99 & 1.14 & 1.06 \\
$\mathrm{~K}$ & 0.95 & 1.01 & 0.98 \\
$\mathrm{P}$ & 1.00 & 1.11 & 1.06 \\
\hline durchschnittlich & 0.97 & 1.09 & 1.03
\end{tabular}

Die Ergebnisse laut Tabelle 14 entsprechen aber nicht der Erwartung, dass nämlich die nach links gerichtete Bewegung phänomenal beträchtlich schneller wäre. Nach Berechnung des geometrischen Mittels, nachdem der Zeitfehler also in Abzug gebracht ist, findet man nur einen Unterschied von $3 \%$. Zwar ist der Unterschied gesichert, $\sigma=3 \%, \sigma_{M}=1 \%$ und $t=3.0$, aber dieser ist doch beträchtlich geringer als man auf Grund der vorherigen Versuche erwarten könnte und gilt überdies nicht für alle Vpn. 
Die Versuche 19a und 19b wurden jetzt in umgekehrter Folge wiederholt und zwar mit $3 \mathrm{Vpn}$. des vorigen Versuches und mit 7 neuen $\mathrm{Vpn}$.

Versuch $19 c$. Der Standard bewog von links nach rechts, die Variabele von rechts nach links.

Versuch $19 d$. Der Standard bewog von rechts nach links, die Variabele von links nach rechts.

TABEILLF 15

Vergleich von zwei horiznntalen Bewegungen in entgegengesetzter Richtung. $V_{\mathrm{s}}: 10 \mathrm{~cm} / \mathrm{sec}$.

\begin{tabular}{c|c|c|c}
\hline Standardrichtung & nach r. & nach 1. & $\begin{array}{c}V_{\mathrm{f}} / V_{l} \\
\text { durchschn. }\end{array}$ \\
\hline Vpn. & $V_{s} / V_{l}$ & $V_{r} / V_{s}$ & \\
\hline vdM & 1.24 & 1.04 & 1.14 \\
$\mathrm{Li}$ & 1.19 & 0.96 & 1.07 \\
$\mathrm{~S}$ & 1.16 & 1.09 & 1.12 \\
Schw & 1.23 & 1.00 & 1.11 \\
$\mathrm{~V}$ & 1.23 & 1.00 & 1.11 \\
$\mathrm{~W}$ & 1.29 & 1.00 & 1.13 \\
$\mathrm{~F}$ & 1.23 & 0.99 & 1.10 \\
$\mathrm{~N}$ & 1.16 & 1.03 & 1.10 \\
$\mathrm{~B}$ & 1.24 & 1.00 & 1.11 \\
$\mathrm{Sp}$ & 1.20 & 0.98 & 1.08 \\
\hline durchschnittlich & 1.22 & 1.01 & 1.11
\end{tabular}

Die Ergebnisse der Tabelle 15 scheinen dem zuvor gefundenen Unterschied zwischen der nach links und der nach rechts gerichteten Bewegung besser zu entsprechen. Nach Abzug des Zeitfehlers gibt es einen phänomenalen Geschwindigkeitsunterschied von $11 \%$. Dieser Unterschied ist gesichert, denn $\sigma=2 \%, \sigma_{M}=0.7 \%$ und $t=15.7$.

Zwischen den neuen Vpn. und den Vpn., die auch an den Versuchen 19a und $19 \mathrm{~b}$ teilnahmen, liegt kein Unterschied vor. Die Frage ist nun, wie soll der Unterschied der Ergebnisse nach Tabelle 14 und 15 (1.03 gegenüber 1.11) erklärt werden und welcher Unterschied ist der richtige? Auf Grund der bei dem Vergleich einer horizontalen und einer vertikalen Bewegung erhaltenen Ergebnisse, scheint es am wahrscheinlichsten, dass die Ergebnisse der Tabelle 15 richtig sind, obgleich ein grösserer Unterschied von ca. $20 \%$ zu erwarten wäre. Der direkte Vergleich zwischen einer nach links und einer nach rechts gerichteten Bewegung ergibt also einen geringeren phänomenalen Geschwindigkeitsunterschied als der indirekte Vergleich, bei dem man die vertikale Bewegung in Beziehung zu den beiden entgegengesetzten 
horizontalen vergleicht und man das Verhältnis zwischen der Geschwindigkeit der nach links und nach rechts gerichteten Bewegungen bei subjektiver Gleichheit berechnen könnte. Im letzten Fa!le ergab sich ein Unterschied in der Grössenordnung von etwa $20 \%$.

Welches Resultat aber am richtigsten ist, das nach Tabelle 14 oder 15, soll einer näheren Prüfung unterzogen werden, und man kann sich abfragen, was geschehen würde, wenn man die Versuche der Tabellen 14 und 15 fortsetzen würde. Mit $2 \mathrm{Vpn}$. wurden nun die Versuche fortgesetzt, und zwar so, dass an einem Tage die Versuche 19a und 19b, am anderen Tage die Versuche 19c und 19d wiederholt wurden. Die Durchschnittswerte von 8 Einstellungen pro Tag für jede der beiden Vpn. sind in Tabelle 16 erwähnt, in der auch die Werte aus den Tabellen 14 und 15 aufgenommen sind.

TABELLE 16

Fortsetzung der Versuche 19 bei zwei Vpn. an verschiedenen Tagen

\begin{tabular}{c|c|c|c|c|c|c}
\hline \multicolumn{2}{c|}{ Versuch 19: } & $\mathrm{a}$ & $\mathrm{b}$ & & $\mathrm{c}$ & $\mathrm{d}$ \\
\hline \multicolumn{2}{c|}{ Standardrichtung } & $\leftarrow$ & $\rightarrow$ & & $\rightarrow$ & $\leftarrow$ \\
\hline Vpn. & $\mathrm{Tag}$ & $V_{r} / V_{l}$ & $V_{r} / V_{l}$ & $\mathrm{Tag}$ & $V / r V_{l}$ & $V_{r} / V_{l}$ \\
\hline \multirow{2}{*}{$\mathrm{Li}$} & 1. & 0.97 & 1.08 & 2. & 1.19 & 0.96 \\
& 3. & 0.97 & 1.08 & 4. & 1.21 & 0.97 \\
& 5. & 0.99 & 1.14 & 6. & 1.17 & 0.97 \\
& 7. & 1.02 & 1.17 & 8. & 1.15 & 1.01 \\
\hline \multirow{3}{*}{$\mathrm{S}$} & 1. & 1.03 & 1.11 & 2. & 1.16 & 1.09 \\
& 3. & 1.03 & 1.12 & 4. & 1.16 & 1.09 \\
& 5. & 1.08 & 1.17 & 6. & 1.17 & 1.09 \\
& 7. & 1.08 & 1.17 & 8. & 1.17 & 1.09
\end{tabular}

Aus Tabelle 16 geht nun hervor, dass - falls die Versuche fortgesetzt werden - das Ergebnis der Versuche a und $b$ dem der Versuche $c$ und $d$ völlig entspricht. Bei Vp. Li sind nach drei Wiederholungen die Quotienten der Versuche a und d bzw. 1.02 und 1.01, die der Versuche $b$ und $c$ bzw. 1.17 und 1.15. Bei Vp. S sind diese Quotienten nach drei Wiederholungen für a und $\mathrm{d}$ bzw. 1.08 und 1.09 und für $\mathrm{b}$ und $\mathrm{c}$ beide 1.17. Die Annahme, dass unter den hier geltenden Verhältnissen die nach links gerichtete Bewegung phänomenal schneller ist als die nach rechts gerichtete (ca. $11 \%$ ), scheint also berechtigt.

Wir wollen jetzt durch einen direkten Vergleich der aszendenten und deszendenten Bewegung prüfen, ob es tatsächlich keinen Geschwindigkeits- 
unterschied zwischen den beiden entgegengesetzten Richtungen der vertikalen Dimension gibt. Dazu wurden die nachstehenden Versuche vorgenommen.

Versuch 20a. Die Bedingungen waren wie in Versuch 19. $V_{s}: 10 \mathrm{~cm} / \mathrm{sec}$. Die Standardbewegung war von oben nach unten; die Variabele von unten nach oben.

Versuch 20b. Die Standardbewegung war von unten nach oben; die Variabele von oben nach unten.

TABELLE 17

Vergleich von zwei vertikalen Bewegungen in entgegengesetzter Richtung. $V_{8}: 10 \mathrm{~cm} / \mathrm{sec}$.

\begin{tabular}{c|c|c|c}
\hline Standardrichtung & $s=d$ & $s=a$ & $\begin{array}{c}\text { durchschn. } \\
V_{d} / V_{a}\end{array}$ \\
\hline Vpn. & $V_{d} / V_{a}$ & $V_{d} / V_{a}$ & 1.02 \\
W & 1.16 & 0.89 & 1.06 \\
Dr & 1.12 & 1.00 & 1.13 \\
K & 1.27 & 1.01 & 1.06 \\
vdS & 1.09 & 1.04 & 0.95 \\
Tr & 0.91 & 0.99 & 0.95 \\
Mi & 0.98 & 0.92 & 1.01 \\
P & 1.00 & 1.02 & 0.97 \\
vB & 1.11 & 0.85 & 0.95 \\
K & 0.99 & 0.91 & 1.01 \\
B & 1.03 & 1.00 & 1.01
\end{tabular}

Der Erwartung gemäss gibt es keinen phänomenalen Geschwindigkeitsunterschied zwischen einer aszendenten und einer deszendenten Bewegung. Das Verhältnis $V_{d} / V_{a}$ nach Abzug des Zeitfehlers beträgt 1.01 (Tab. 17).

$\mathrm{Da}$ aus dem Vergleich der Geschwindigkeiten in den beiden horizontalen Richtungen hervorgegangen war, dass die Grösse des ermittelten Geschwindigkeitsunterschiedes eventuell von der Expositionsfolge abhängig sein könnte, wurden die Versuche 20a und b auch noch in umgekehrter Folge wiederholt.

Versuch 20 c. Die Standardbewegung war in aszendenter, die Variabele in deszendenter Richtung.

Versuch 20d. Die Standardbewegung war in deszendenter, die Variabele in aszendenter richtung. 
TABELLE 18

ich von zwei vertikalen Bewegungen in entgegengesetzter Richtung. $V_{8}: 10 \mathrm{rm} / \mathrm{sec}$.

\begin{tabular}{c|c|c|c}
\hline \hline Standardrichtung & $s=a$ & $s=d$ & $\begin{array}{c}\text { durchschn. } \\
V_{d} / V_{a}\end{array}$ \\
\hline Vpn. & $V_{d} / V_{a}$ & $V_{d} / V_{a}$ & 1.03 \\
dJ & 0.94 & 1.14 & 0.97 \\
F & 1.00 & 0.93 & 1.00 \\
dV & 1.00 & 1.00 & 1.01 \\
S & 0.95 & 1.07 & 0.99 \\
vdM & 1.02 & 0.97 & 0.95 \\
Bo & 1.00 & 0.90 & 0.89 \\
vBa & 0.87 & 0.92 & 0.98 \\
Dr & 0.87 & 1.10 & 0.97 \\
Kl & 0.88 & 1.07 & 1.03 \\
vBr & 1.00 & 1.06 & 0.98
\end{tabular}

Der Durchschnittswert des Verhältnisses $V_{d} / V_{a}$ laut Tabelle 18 beträgt 0.98. Die Ergebnisse der Tabellen 17 und 18 kann man als übereinstimmend betrachten und die Annahme scheint berechtigt, dass es unter den hier geltenden Verhältnissen keinen phänomenalen Geschwindigkeitsunterschied zwischen einer aszendenten und einer deszendenten Bewegung gibt.

Bei der Projizierung einer horizontalen und einer vertikalen Bewegung an der Decke (Versuch 17) scheint ein phänomenaler Geschwindigkeitsunterschied zwischen den Bewegungen in entgegengesetzter Richtung der Vertikalen, bzw. den Bewegungen, parallel der Längenachse des Körpers, vorzuliegen. Dies wollen wir jetzt näher prüfen, indem wir die Geschwindigkeiten der Bewegungen in diesen beiden Richtungen miteinander vergleichen.

Versuch 21. Die Bewegungen wurden auf die Decke projiziert; die Vp. lag auf einem Sofa; die Verhältnisse waren wie in Versuch 17. $V_{s}: 10 \mathrm{~cm} / \mathrm{sec}$.

a) Die Standardbewegung verlief in aszendenter (sich von der Vp. entfernender), die Variabele in deszendenter (sich der Vp. nähernder) Richtung;

b) Die Standardbewegung verlief in deszendenter (sich der Vp. nähernder), die Variabele in entgegengesetzter Richtung.

Aus Tabelle 19 geht also tatsächlich hervor, dass die aszendente, bzw. die sich von der $\mathrm{Vp}$. entfernende Bewegung, phänomenal beträchtlich schneller ist als die deszendente, bzw. die sich der $\mathrm{Vp}$. nähernde Bewegung. 
TABELLE 19

Vergleich von an der Decke projizierten Bewegungen in entgegengesetzter Richtung. Beobachtung in liegender Haltung. $V_{s}: 10 \mathrm{~cm} / \mathrm{sec}$.

\begin{tabular}{c|c|c}
\hline \hline Standardrichtung & $s=a$ & $s=d$ \\
\hline Vpn. & $V_{d} / V_{a}$ & $V_{d} / V_{a}$ \\
\hline $\mathrm{V}$ & 1.58 & 1.45 \\
vdS & 1.41 & 1.42 \\
$\mathrm{~B}$ & 1.40 & 1.46
\end{tabular}

Versuch 22. Wie Versuch 21; die Vp. sass aber jetzt aufrecht; die bewegenden Punkte erschienen schräg vor ihr an der Decke.

TABELLE 20

Bewegungen an der Decke. Beobachtung aufrecht sitzend

\begin{tabular}{c|c|c}
\hline Standardrichtung & $s=d$ & $s=a$ \\
\hline Vpn. & $V_{d} / V_{a}$ & $V_{d} / V_{a}$ \\
\hline $\mathrm{P}$ & 1.12 & 1.10 \\
$\mathrm{R}$ & 1.02 & 0.98 \\
$\mathrm{~W}$ & 1.09 & 0.98
\end{tabular}

Nach Tabelle 20 scheint jetzt wenig oder gar kein Unterschied zwischen der aszendenten und der deszendenten Bewegung vorzuliegen.

Aus den bisherigen Versuchen ersehen wir, dass die phänomenale Geschwindigkeit von Bewegungen in den verschiedenen Richtungen unseres Wahrnehmungsraumes bei gleicher objektiver Geschwindigkeit nicht immer dieselbe ist. Eines der überraschendsten Ergebnisse ist, dass der Geschwindigkeitsunterschied zwischen einer horizontalen und einer vertikalen Bewegung, der von Brown genannt und seitdem überall in der Literatur erwähnt wird, nur gilt, falls die horizontale Bewegung von links nach rechts verläuft. Die Bewegungen nach links und nach rechts weisen einen Unterschied von ca. $11 \%$ auf, während die Bewegung von rechts nach links schneller gesehen wird. Diese Ergebnisse lassen sich schwer erklären, da Rechts- und Linkshändigkeit, bzw. Rechts- und Linksäugigkeit, keinen Einfluss darauf hat.

Bewegungen in verschiedenen Richtungen haben aber auch eine gewisse Bedeutung für uns, die mit unserer Organisation und unserem Orientierungssystem in Zusammenhang steht. Dass die beiden Richtungen 
der horizontalen Dimension eine verschiedene Bedeutung haben, wollen wir in den beiden nachstehenden Versuchen untersuchen. Die Anzahl Vpn. betrug in beiden Fällen 42 .

Versuch 23. Die Vpn. wurden gebeten, ein ausfahrendes Schiff zu zeichnen.

Versuch 24. Der Auftrag lautete: Stellen Sie sich vor, dass Sie spazieren gehen und dass Sie unterwegs Ihrem Freunde begegnen. Zeichnen Sie nun ein Bild Thres Freundes.

In Tabelle 21 sind die Ergebnisse dieser beiden Versuche aufgezeichnet.

TABELLE 21

Bedeutung der entgegengesetzten Richtungen in der horizontalen Dimension bei Zeichenversuchen

\begin{tabular}{l|r|r|r|r}
\hline & \multicolumn{4}{|c}{ gezeichnete Richtung } \\
\cline { 2 - 5 } & \multicolumn{3}{|c|}{$\rightarrow$} & \multicolumn{2}{c}{$\leftarrow$} \\
\cline { 2 - 5 } & $\mathrm{n}$ & $\%$ & $\mathrm{n}$ & $\%$ \\
\hline ausfahrendes Schiff & 32 & 76 & 10 & 24 \\
Person, der man begegnet & 7 & 17 & 35 & $\mathbf{8 3}$
\end{tabular}

27 der 42 Personen oder $64 \%$ zeichneten das ausfahrende Schiff nach rechts und die Person, der man begegnet, nach links gerichtet; 2 Personen oder $5 \%$ zeichneten beide in entgegengesetzter Richtung; 13 Personen oder $31 \%$ zeichneten das Schiff und die Person in der gleichen Richtung. Von diesen letzten muss man annehmen, dass die Bewegungsrichtung für sie keine Bedeutung hat, oder dass sie sich nicht in die Aufgabe vertieft haben und die Richtung ihrer Zeichnung vom Zufall bestimmt wurde. Auch in den beiden erstgenannten Gruppen werden sich vermutlich einige Personen befinden, deren Ergebnisse vom Zufall bestimmt sind, so dass der Umstand, dass bei 2 Vpn. ein Ergebnis, der Erwartung entgegengesetzt, gefunden wird, keine Bedeutung hat. Es lag kein Unterschied zwischen Rechts- und Linkshändigkeit vor; die beiden Vpn., die ein entgegengesetztes Resultat lieferten, waren rechtshändig.

Der Unterschied zwischen den 27 Vpn., für die die ausfahrende Bewegung nach rechts gerichtet ist, die nähernde nach links, und die $2 \mathrm{Vpn}$. für die diese Richtungen umgekehrt verlaufen, ist wesentlich gesichert $(\mathrm{t}=5.7 ; \mathrm{P}<0.001)$.

Aus diesen Versuchen scheint die Annahme berechtigt, dass im allgemeinen eine nach rechts gerichtete Bewegung die Bedeutung einer Entfernung, eine nach links gerichtete Bewegung die Bedeutung einer Näherung hat. 


\section{ZUSAMMENFASSUNG DER ERGEBNISSE}

1. Bei einer Feldgrösse von $46.2 \times 46.2 \mathrm{~cm}$ und $30 \times 30 \mathrm{~cm}$ und einem Punktdurchmesser von bzw. 40 und $30 \mathrm{~mm}$, gibt es einen Unterschied in phänomenaler Geschwindigkeit zwischen einer vertikalen und einer nach links gerichteten Bewegung (s. Tabellen 1, 2 und 3).

a. Es scheint von der Geschwindigkeit der bewegenden Punkte abhängig zu sein, ob die horizontale oder die vertikale Bewegung schneller ist.

$b$. Es hat den Anschein, dass es zwei Typen Vpn. gibt: der eine sieht bei einer Geschwindigkeit von $5 \mathrm{~cm} / \mathrm{sec}$. die nach links gerichtete, horizontale Bewegung bei gleicher objektiver Geschwindigkeit schneller, bei einer Geschwindigkeit von $20 \mathrm{~cm} / \mathrm{sec}$. die vertikale Bewegung schneller; bei dem anderen Typ ist dieses Verhältnis gerade umgekehrt.

2. Bei einer Feldgrösse von $15 \times 15 \mathrm{~cm}$ und einem bewegenden Punkt von $14 \mathrm{~mm} \emptyset$, ist mit freien Augenbewegungen die phänomenale Geschwindigkeit der vertikalen Bewegung ca. $30 \%$ grösser als die der nach rechts gerichteten, horizontalen Bewegung.

Hinsichtlich der nach links gerichteten, horizontalen Bewegung gibt es wenig oder gar keinen Unterschied (Tab. 4 und 5).

3. Eine nach links gerichtete, horizontale Bewegung ist bei gleicher objektiver Geschwindigkeit phänomenal ca. $11 \%$ schneller als eine nach rechts gerichtete (Tab. 15).

4. Es gibt keinen Unterschied in phänomenaler Geschwindigkeit zwischen den beiden Richtungen der vertikalen Dimension (Tab. 17 und 18).

5. Es macht keinen wesentlichen Unterschied ob die Beobachtung monokular oder binokular ist.

6. Unter Fixation der Mitte der beiden Bewegungsbahnen wird der phänomenale Geschwindigkeitsunterschied zwischen der vertikalen und der horizontalen Bewegung beträchtlich herabgesetzt. Der Unterschied zwischen einer nach rechts und einer nach links gerichteten Bewegung wird bei strenger Fixation aufgehoben (Tab. 7).

7. In liegender Haltung im Dunkeln besteht eine Unsicherheit über die Orientierung nach den Hauptdimensionen unseres Wahrnehmungsraumes. Besonders am Anfang ist die Labilität der Orientierung gross, wobei bald die alte Orientierung nach der objektiven Vertikalen, bald die gemäss der Stellung des Körpers überwiegt (Tab. 8).

8. In liegender Haltung unter schwacher Beleuchtung wird die normale Orientierung leichter beibehalten als im Dunkeln (Tab. 9). 
9. Bei einer Kopfhaltung von $45^{\circ}$ seitwärts nach links oder nach rechts, werden die horizontale und die vertikale Bewegung überwiegend symmetrisch unter einem Winkel von $45^{\circ}$ hinsichtlich des Kopfes wahrgenommen, wobei also die Kopfhaltung als Referenzsystem dient. Es lag jetzt kein Unterschied in phänomenaler Geschwindigkeit vor (Tab. 10 und 11).

10. Wenn die Bewegungen an der Decke projiziert werden, etwas hinter der Vp., die in liegender Haltung die Bewegungen beobachtet, gibt es einen beträchtlichen Unterschied in phänomenaler Geschwindigkeit zwischen der horizontalen und der vertikalen Bewegung. Uberdies ist jetzt die aszendente Bewegung phänomenal schneller als die deszendente (Tab. 12 und 19).

11. Wenn die $\mathrm{Vp}$. aufrecht sitzend die Bewegungen schräg vor ihr an der Decke wahrnimmt, gibt es wenig oder gar keinen Unterschied in phänomenaler Geschwindigkeit zwischen der sich nähernden und der sich entfernenden Bewegung. Für einige Vpn. ist die horizontale Bewegung phänomenal schneller als die vertikale, bzw. sagittale Bewegung, für andere macht es keinen Unterschied oder ist die vertikale bzw. sagittale Bewegung schneller. Die interindividuellen Differenzen sind gross und hängen wahrscheinlich zusammen mit der Weise, wie man die Bewegungen lokalisiert, d.h. in einer frontalparallelen Fläche oder an der Decke. Die Vpn. fühlten sich ziemlich desorientiert (Tab. 13 und 20).

\section{Versuch zur Deutung der Resultate}

Im ersten Paragraphen haben wir uns die Frage vorgelegt, ob der phänomenale Geschwindigkeitsunterschied zwischen einer objektiv vertikalen und horizontalen Bewegung bei Veränderung der Haltung des Kopfes oder der Stellung des Körpers beibehalten bleibe? In den vorherigen Versuchen haben wir gesehen, dass dies nicht der Fall ist. Ebensowenig tritt in liegender Haltung, wenn die Lage des Körpers also um $90^{\circ}$ gedreht ist, eine Umkehrung dieses Unterschiedes auf, was der neuen Lage des Körpers und dem Augenstand entsprechen würde.

Wenn auch physiologische Veränderungen Motive für die Erfassung des Wahrnehmbaren liefern ${ }^{1}$, so ist doch die Bedeutung der Hauptrichtungen

1 Die Versuche von R. P. Mesker (De optische localisatie onder invloed van optische en houdingsfactoren, Amsterdam, 1953) zeigen eine Beziehung der optischen Lokalisation zur Körperhaltung. Der Autor basiert sich auf der innervationstonustheorie von C. O. Roelofs (Die optische Lokalisation, Arch. Augenheilk. 109, 1936) 
des visuellen Wahrnehmungsraumes, bis zu einem gewissen Grade, von dem Entwurf, den wir wählen, abhängig. Wie könnte man z.B. physiologisch erklären, dass die Geschwindigkeitstäuschung sich völlig umkehrt, wenn man in liegender Haltung die objektiv horizontale Bewegung als eine vertikale auffasst, d.h. in dem sich objektiv horizontal bewegenden Punkt einen fallenden oder steigenden Punkt sieht, während dies bei unvoreingenommener, naiver Beobachtung keineswegs der Fall ist?

Unser visueller Wahrnehmungsraum besitzt in der horizontalen, der vertikalen und der sagittalen Dimension ein Referenzsystem, auf das wir unsere Wahrnehmungen beziehen. Der Wert, den die drei Dimensionen für dieses Referenzsystem haben, ist aber nicht gleichwertig, sondern u.a. davon abhängig, ob wir ein Objekt in Ruhe oder in Bewegung, im Hellen oder im Dunkeln beobachten. Vor allem aber wird deren Wert dadurch bestimmt, dass wir selber daran teilhaben, selber dieses Referenzsystem mitkonstituieren und ihm eine bestimmte Bedeutung gewähren. Die Lage eines Gegenstandes im Raum wird nicht nur von den drei Dimensionen des objektiven Raumes als Referenzsystem bestimmt, aber diese Lage erhält erst ihre Konsolidation durch die Bedeutung, die ich dem Referenzsystem verleihe, und zwar aus der Relation, die zwischen mir und dem Referenzsystem besteht.

Ob wir eine Bewegung als horizontal oder vertikal auffassen, wird nicht nur von der Horizontalen oder der Vertikalen des objektiven Raumes bestimmt, ebensowenig nur durch die Lage, die der Körper oder die Augen hinsichtlich der Richtung der Bewegung einnehmen, sondern durch die Bedeutung, die das Referenzsystem, das zusammen mit mir eine Einheit bildet, für mich hat. Wenn die Lage des Körpers bezüglich der objektiven Vertikalen verändert wird, verändert damit nicht im gleichen Masse die Lage der optischen Vertikalen. Was ich horizontal oder vertikal nenne, korrespondiert nicht nur mit der Lage meines Körpers oder meiner Augen; fällt also nicht zusammen mit den horizontalen, bzw. vertikalen Retinameridianen. Ebensowenig behauptet sich die ursprüngliche Lage. Welcher Dimension ich in liegender Haltung die Bedeutung vertikal gewähre, wird hauptsächlich von den anderen Motiven, die meinen Raum mitkonstituieren, bedingt werden: die Gegenstände, die sich darin befinden, die für mich eine Bedeutung haben, in der die Bedeutung des ursprünglichen Referenzsystems mitgegeben ist und die jetzt ihrerseits als Referenzsystem für meine Orientierung dienen. Daher wird man im Dunkeln die ursprünglich objek-

und erklärt die Stellung der optischen Vertikalen unter Einfluss von optischen und Haltungsfaktoren aus Anderungen des reflektorischen Blicktonus und des reflektorischen Innervationstonus der Körpermuskulatur. 
tive Vertikale vorzugsweise als mehr horizontal gerichtet auffassen. Im Hellen behauptet die objektive Vertikale sich leichter als optische Vertikale, da die Gegenstände, die ich wahrnehme, dann als Hauptmotive zur Konstitution meines Raumes funktionieren und die ursprüngliche Orientierung stabilisieren.

Eine gewisse Labilität in der Orientierung wird sich, besonders im Anfang nach einer Lageänderung des Körpers, geltend machen, wenn auch kein festes Referenzsystem mit einer neuen Bedeutung hergestellt ist. Ich besitze aber innerhalb gewisser Grenzen die Freiheit, an dieser Sinnverleihung einen aktiven Anteil zu nehmen. Die phänomenale Richtung der Bewegungen wird bestimmt durch die Bedeutung, die ich ihr verleihe, ungeachtet ob ich mich dabei mehr passiv oder aktiv verhalte.

Aus den Versuchen im Paragraphen 4 ergab sich, dass die nach links gerichtete Bewegung bei objektiver Gleichheit schneller gesehen wird als die nach rechts gerichtete. Weiter sahen wir, dass man der nach rechts gerichteten Bewegung die Bedeutung von Ausfahren oder Entfernen, der nach links gerichteten die von Begegnen, Nähern beimisst. Die Bedeutungen unserer Welt und unsere Organisation sind immer korrelativ aufeinander bezogen. Den Raum als bewohnbaren Raum, als einen Raum in dem die Dinge ihren Platz und ihre Bedeutung haben, konstituiere ich, indem ich mich in ihn begebe, und ich vollziehe virtuell die Bewegung mit, die ich wahrnehme. Nun ist der Mensch ein expansives Wesen, ein Wesen, das ,ausfährt" und nicht an einem Ruhepunkte verweilt. Er befindet sich unterwegs und seine natürliche Bewegungsrichtung - und diese ist zugleich die Richtung, in der er sich orientiert - scheint, insofern es die horizontale Dimension betrifft, nach rechts gerichtet zu sein. Ich möchte nun die Hypothese vorschlagen, dass wir dadurch, dass wir uns unterwegs befinden und zwar nach rechts gerichtet, die. Geschwindigkeit einer Bewegung in derselben Richtung als unsere Orientierungsrichtung unterschätzen und diejenige der Bewegung, die uns phänomenal begegnet, ïberschätzen. ${ }^{2}$

Die Menschheit hat von alters her einen verschiedenen Sinn mit rechts und links verbunden. Diese Verschiedenheit kommt noch in zahlreichen Gebräuchen und Ausdrücken zur Ausserung. Meistens wird mit rechts der Sinn des Guten verbunden, bisweilen aber auch mit links. Hierin besteht

2 Ich befasse mich momentan mit einer Anzahl Versuchen, um dicsen Zusammenhang näher zu begründen. Es hat z.B. den Anschein, dass die Kopfbewegungen nach rechts leichter und schneller seien als die nach links. Die Versuche befinden sich aber noch nicht in einem Stadium, dass deren Resultate veröffentlicht werden könnten. 
wenig Ubereinstimmung. Auch im Altertum findet man in zahlreichen kultischen Gebräuchen diesen Unterschied zwischen rechts und lirks. Bachofen weist darauf hin, dass in alten matriarchalen Kulturen die linke Hälfte des Körpers die bevorzugte Seite war, während dagegen in patriarchalen Kulturen die rechte Seite den Vorzug hatte. Links wird mit dem Weiblichen, Passiven, rechts mit dem Männlichen, Aktiven verbunden. Im Indogermanischen heisst die rechte Hand männlich, die linke weiblich (Thurnwald).3

Auch in der psychoanalytischen Literatur hat man dem Problem RechtsLinks Aufmerksamkeit gewidmet. Rechts ist bei den Patienten meistens ein Symbol des Guten, doch auch wohl links. Nach Rümke + sei hier jedoch nicht primär die Schätzung vom Guten oder vom Bösen, diese Sinnverleihung sei sekundär und von der Lebensgeschichte abhängig, sondern handele es sich um die Würdigung des Weiblichen und des Männlichen, wobei links das Weibliche, rechts das Männliche vertrete. Bei Linkshändern wäre dieser Unterschied umgekehrt.

Die Asymmetrie, die wir in der Schätzung der Geschwindigkeit nach rechts und links gefunden haben, ist jedoch nicht von Rechts- oder Linkshändigkeit abhängig, sei es, dass vermutlich wohl ein geringer Unterschied vorliegt und die Täuschung bei Rechtshändern etwas grösser ist. Mit einer Unterscheidung in eine männliche oder aktive und eine weibliche oder passive Hälfte unserer Organisationsstruktur, die von Links- und Rechtshändigkeit, bzw. Links- und Rechtsäugigkeit abhängig wäre, ist jedoch der Kern unseres Problems nicht berührt. Für die Beurteilung der Geschwindigkeit in der horizontalen Dimension scheint mir primär unsere eigene Bewegungsgerichtetheit bestimmend zu sein, die man vielleicht als eine jedem Verhalten vorgegebene Orientierung unseres In-der-Welt-Seins zu verstehen hat. 5

In der vertikalen Dimension bestehen keine Differenzen in phänomenaler Geschwindigkeit zwischen den beiden entgegengesetzten Richtungen. Dies wird deutlich, wenn wir erwägen, dass unsere Stellung hinsichtlich der Horizontalen und der Vertikalen radikal anders ist. Kraft unserer Organisation und der Gravitation, der wir unterworfen sind, sind wir von oben nach unten gcrichtet, doch wir haben in dieser Gerichtetheit den Endpunkt als Ruhepunkt erreicht. Dadurch dass wir in der vertikalen Dimension einen

3 Zit. nach H. C. Rümke: Studies en Voordrachten, 1943. S. 371.

4 H. C. Rümke: Rechts en Links in de Psychopathologie, Studies en Voordrachten, 1943.

5 Weitere Untersuchungen, etwa bei Völkern, deren Schrift von rechts nach links ausgeführt wird, könnten möglicherweise ein neues Licht auf dieses Problem werfen. 
Ruhepunkt erreicht haben, ist die Bewohnbarkeit unseres Raumes gesichert, und sind wir imstande, unsere Stabilität zu behaupten. In der horizontalen Ebene dagegen befinden wir uns unterwegs. Da wir uns in vertikaler Richtung phänomenal nicht bewegen, wird die Geschwindigkeit in aufwärtser Richtung nicht überschätzt.

Wenn wir in liegender Haltung die Bewegungen über und schräg hinter uns beobachten, gibt es wohl einen Unterschied in phänomenaler Geschwindigkeit zwischen der nach oben und der nach unten gerichteten Bewegung, wie wir ersehen haben. $\mathrm{Da}$ ich die Bewegung, die ich wahrnehme, virtuell mit vollziehe, hat meine Bewegung in der abwärtsen Richtung phänomenal den Endpunkt als Ruhepunkt noch nicht erreicht. Dies bildet für uns eine unnatürliche Situation, eine Situation, deren Stabilität aufgehoben ist und die nicht bewohnbar ist. Dies möge auch aus dem Umstand hervorgehen, dass in dieser Situation die Konstanzphänomene in der Organisation unserer Wahrnehmung beträchtlich verringert sind: Formund Grössenkonstanz sind grösstenteils aufgehoben.

Eine weitere Unterstützung für die hier entwickelte Hypothese dürfte der Umstand geben, dass bei strenger Blickfixation der Unterschied in phänomenaler Geschwindigkeit zwischen einer Bewegung nach rechts und nach links ausgeglichen ist.

Die Hypothese, dass unsere Orientierungsrichtung in der vertikalen Dimension von oben nach unten läuft, könnte man vermutlich als im Einklang mit der Bodenständigkeitshypothese von De Boer ${ }^{6}$ und den Auffassungen Benussis betrachten, nach dem ambigue Figuren vorzugsweise gesehen werden, als ob man sie von oben herab betrachtet. Nach Corkill und Lythgoe 7 wären die Augenbewegungen von oben nach unten leichter und schneller als von unten nach oben. Auch dies könnte im Einklang mit unserer natürlichen Gerichtetheit von oben nach unten sein.

Der phänomenale Geschwindigkeitsunterschied zwischen einer horizontalen und einer vertikalen Bewegung ist, unter Beibehaltung des Prinzips, dass die Geschwindigkeit in der Richtung der natürlichen Orientierung unterschätzt wird, leicht zu erklären.

Dem hier entwickelten Gedankengang entsprechend, sind zwischen Bewegungen in verschiedenen Richtungen qualitative Differenzen zu erwarten. Befragt man die Vpn. ob sie zwischen zwei Bewegungen in verschiedenen Richtungen qualitative Verschiedenheiten angeben könnten, so

- De Boer: Uber umkehrbare Zeichnungen, Arch. ges. Psychol. 18, 1910.

7 Corkill and Lythgoe: Some experiments on eye-movements. Brit. J. Ophthal. 13, 1929. 
wurde einstimmig die vertikale Bewegung als eine aktivere gegenüber der horizontalen beurteilt, was der subjektiven Erlebung einer grösseren Geschwindigkeit entspricht.

Die Verschiedenheit zwischen der nach rechts und der nach links laufenden Bewegung war schwieriger zu beschreiben. In diesem Falle wurden zwei Differenzen erwähnt. Die eine war, dass die Bewegung nach links mehr Widerstand hervorrief und dadurch subjektiv ein grösseres Aktivitätsgefühl bewirke. Für einige Linkshänder war es aber die Bewegung nach rechts, die ein Gefühl grösseren Widerstandes hervorrief, so dass hiermit nicht die wesentliche Differenz gekennzeichnet ist. Die andere Verschiedenheit, die auch für Linkshänder galt, war, dass die nach rechts gerichtete Bewegung den Eindruck einer passiven, gleichmässig weiterfliessenden Bewegung machte, während die nach links gerichtete Bewegung einen abrupteren Charakter hatte.

Von der nach oben und nach unten gerichteten Bewegung gelang es nicht von den Vpn. eine Beschreibung irgendeiner Differenz zu erhalten. Auf einem grösseren Felde und bei einer Geschwindigkeit von $5 \mathrm{~cm} / \mathrm{sec}$. wurde aber eine Asymmetrie zwischen den beiden Hälften des Feldes wahrgenommen: die Bewegung über die untere Hälfte war phänomenal schneller als über die obere Hälfte. Unter diesen Umständen hätte es wahrscheinlich wohl einen Unterschied in phänomenaler Geschwindigkeit zwischen der aszendenten und der deszendenten Bewegung gegeben. Dies wurde aber nicht geprüft. Die Absicht war, die Versuche unter möglichst reduzierten Verhältnissen (im Dunkeln, kurze Expositionszeit) vorzunehmen, da dies die günstigsten Bedingungen schafft, die ursprünglichen Orientierungstendenzen zu zeigen.

Nachdem ich meine Versuche beendigt hatte, kam mir das Buch von Martti Takala: "Asymmetries of the visual space" (Helsinki 1951), in die Hände. Die von Takala gefundenen Asymmetrien in der Organisation unseres visuellen Wahrnehmungsraumes entsprechen den in meiner Untersuchung gefundenen Bewegungsasymmetrien. Auf Grund zahlreicher Versuche über Lokalisierung, Erinnerung und Wiedererkennung kommt er zur Folgerung, dass unser optischer Wahrnehmungsraum asymmetrisch ist und die linke und obere Hälfte bevorzugt werden. Auch in der Weise, in der wir unsere Wahrnehmungen organisieren, gibt es Vorzugsrichtungen: ein Objekt in Ruhe wird leichter wahrgenommen und wiedererkannt, wenn es nach links oder nach oben gerichtet ist als nach rechts bzw. nach unten. Die Vorzugsrichtungen eines statischen Objektes sind also horizontal von rechts nach links, vertikal von unten nach oben und sagittal auf uns hingerichtet. Bei der Beurteilung der Geschwindigkeit einer Bewegung ist die 
Asymmetrie derjenigen der statischen Objekte gleichsam entgegengesetzt. Bewegung nach links, nach oben und auf uns hingerichtet, werden überschätzt. Takala betrachtet dies im Einklang mit den Richtungen der spontanen Augenbewegungen, die von links nach rechts, von oben nach unten leichter seien und leichter divergieren als konvergieren sollten. Dementsprechend sei es leichter einem Objekt. das sich in diesen Richtungen bewegt, zu folgen, weshalb eine Bewegung in entgegengesetzter Richtung überschätzt wird. Diese Úberschätzung wäre dann eine Folge des Umstandes, dass hierzu eine grössere Muskelenergie benötigt würde, womit das Problem auf eine physiologische Basis reduziert wäre. M.E. ist es aber schwerlich anzunehmen, dass bei der Beobachtung einer Bewegung über ein Feld von $15 \mathrm{~cm}$ eine Differenz in Quantum Muskelenergie, für das Folgen einer Bewegung nach links oder nach rechts erforderlich, diesen grossen phänomenalen Geschwindigkeitsunterschied erklären könnte. Nach Corkill und Lythgoe gäbe es keine Differenz in der Geschwindigkeit der Augenbewegungen nach links und nach rechts. Takala mcint aber, auf Grund seiner Versuche, eine geringe Andeutung erhalten zu haben, dass die Augenbewegungen nach rechts leichter und schneller seien. Bei der Asymmetrie, die in der Organisation der Wahrnehmung von Objekten in Ruhe auftritt, sollten die Augenbewegungen keine Rolle spielen.

Von Takala werden für die Wahrnehmung von Bewegungen also gleichfalls Vorzugsrichtungen angenommen, welche von links nach rechts, von oben nach unten und sagittal in entfernender Richtung laufen müssten. In diesen Richtungen wird die Geschwindigkeit eines Objektes unterschätzt. In seinen Versuchen über die Beurteilung der Geschwindigkeit erlangt Takala aber andere Resultate als von mir gefunden werden. Nun besteht in der Versuchsanordnung eine bedeutende Differenz, die den Unterschied in Ergebnissen möglicherweise bedingt hat. Meine Versuche wurden unter möglichst reduzierten Verhältnissen vorgenommen. In Takalas Versuchen dagegen waren die bewegenden Objekte Quadrate von $10 \times 10 \mathrm{~cm}$, die sich im Hellen mit einer Geschwindigkeit von $8 \mathrm{~cm} / \mathrm{sec}$. gegen einen schwarzen Hintergrund über einen Abstand von $1.20 \mathrm{~m}$ bewegten. Der Beobachtungsabstand war $6 \mathrm{~m}$. Die Anzahl Vpn. war 10; von jeder wurde 6 Einstellungen erhalten. Takala bemerkt hierzu aber selber, dass infolge der mangelhaften Apparatur die Ergebnisse nicht als endgültig zu betrachten seien, ausgenommen den in der sagittalen Dimension geflindcnen, wo die Differenzen bedeutend sind. Seine Ergebnisse sind:

a. Es liegt kein Unterschied in phänomenaler Geschwindigkeit vor zwischen einer Bewegung nach rechts und nach links.

$b$. Die Geschwindigkeit der aszendenten Bewegung gegenüber der 
deszendenten wird überschätzt. Der Unterschied sei statistisch auf dem Niveau der $1 \%$-Verlässlichkeit gesichert $(t=3.3)$.

c. In der sagittalen Dimension wird die sich nähernde Bewegung gegenüber der sich entfernenden überschätzt. Der Unterschied sei gesichert auf dem Niveau der $1 \%$-Verlässlichkeit $(t=4.3)$.

Anlässlich $a$ kann man bemerken, dass dieses Resultat nicht völlig seiner Erwartung, gegründet auf die von ihm gefundene Asymmetrie in der Organisation unserer Wahrnehmung von statischen Objekten, entspricht. Zwar wäre, naci Takala, die Asymmetrie der horizontalen, im Gegensatz zu der der vertikalen Dimension, unter weniger reduzierten Verhältnissen geringer. Da seine Versuche im Hellen und mit einer Expositionszeit von $15^{\prime \prime}$ vorgenommen wurden, die meinigen dagegen im Dunkeln mit einer Expositionszeit von 1.5", ist es sehr gut möglich, dass hierdurch der Unterschied in den Ergebnissen verursacht worden ist. Der Einfluss der Erfahrung wird wahrscheinlich eben in der horizontalen Dimension wohl kompensierend auf die ursprüngliche 8 Asymmetrie wirken.

$A d b$. Die steigende Bewegung wird überschätzt, was der Asymmetrie entspricht, die er in der vertikalen Dimension in den Organisationsphänomenen unserer Wahrnehmung von Objekten in Ruhe findet. Takala erwähnt, dass der Unterschied auf dem Niveau der $1 \%$-Verlässlichkeit gesichert sei. Dies ist aber nicht richtig: die Sicherheitsschwelle ist nur $5 \%$. Da zudem die Apparatur als wenig verlässlich von ihm betrachtet wurde, ist dieser Unterschied wohl einigermassen fragwürdig.

Takala teilt nachstehende Daten mit:

Der Standard bewog sich nach unten, die Variabele nach oben. Die Zeitdifferenz war, über einen Abstand von $1.40 \mathrm{~m}$ gemessen, durchschnittlich $V_{1}-S=1.8^{\prime \prime}$. Daraufhin wurde die Reihenfolge der Bewegungen umgekehrt und war der Zeitunterschied $V_{2}-S=-0.2^{\prime \prime}$. Er nimmt nun die Differenz zwischen $V_{1}$ und $V_{2}$, die 2" beträgt und basiert hierauf die $t$-Berechnung. Da der Effekt der Täuschung in $V_{1}$ und $V_{2}$ entgegengesetzt ist, macht $2 "$ also den doppelten Wert des tatsächlichen Unterschieds zwischen einer steigenden und einer fallenden Bewegung aus. Nun besteht kein Bedenken dagegen, diesen doppelten Wert in Anrechnung zu bringen, vorausgesetzt dass man dann darauf achtet, dass es wahrscheinlich eine grosse negative Korrelation zwischen den beiden Methoden gibt. Der theoretische Wert des Korrelationskoeffizienten beträgt -1 , und man darf annehmen, dass der empirische Wert gross ist, umsomehr da auch die Streuungen in beiden Fällen gleich sind, nämlich $1.2^{\prime \prime}$. Takala berücksichtigt die Korrelation jedoch nicht. Als durchschnitt-

8 Wenn ich von einer ursprünglichen Richtungstendenz rede, ist hiermit. nicht gemeint, dass diese für Kinder ebenfalls oder vielleicht stärker gelte. Es ist sehr gut möglich, dass dies nicht der Fall ist. Dies bedarf aber einer näheren Untersuchung. 
lichen Unsicherheitswert über beide Methoden erwähnt er $0.6^{\prime \prime}$ und berrechnet $t$ aus $2^{\prime \prime}: 0.6^{\prime \prime}=3.3$. Da er nur die Durchschnittsdifferenz der $10 \mathrm{Vpn}$. erwähnt, ist der Korrelationskoeffizient nicht zu berechnen, aber $t$ wird bestimmt kleiner sein als 3.3. Der theoretische Wert ist $t=2^{\prime \prime}: 0.8^{\prime \prime}=2.5$. Da der kritische Bruch bei 9 Freiheitsgraden auf dem Niveau der $1 \%$-bzw. $5 \%$-Verlässlichkeit resp. 3.25 und 2.26 beträgt, ist der Unterschied also nur auf dem Niveau der $5 \%$-Verlässlichkeit gesichert.

Da die Streuungen für jede Methode 1.2" betragen und die Grösse der Täuschung durchschnittlich 1" ist, gilt die Täuschung auch nicht für alle Vpn., sondern entweder nur etwa für die Hälfte, oder zwar für die Mehrheit, aber dann gibt es sogar einige Vpn. mit entgegengesetzter Täuschung. Da Takala die individuellen Werte nicht erwähnt, ist dies nicht festzustellen.

Ad.c. Hierfür gilt mutatis mutandis dieselbe Auseinandersetzung. Der theoretisch zu erwartende Wert von $t$ ist 3.09 , so dass es fragwürdig ist, ob der Unterschied auf dem Niveau der $1 \%$-Verlässlichkeit gesichert ist.

Dass in der sagittalen Dimension die sich nähernde Bewegung phänomenal überschätzt wird, können wir als im Einklang mit unserer Hypothese betrachten.

Vergleichen wir meine Resultate mit denjenigen Takalas, so möchte ich zur folgenden Konklusion kommen.

Wir besitzen im Raum Vorzugsrichtungen, in denen wir uns orientieren. Diese verlaufen horizontal von links nach rechts, vertikal von oben nach unten und sagittal in entfernender Richtung. Da wir existentiell auf die Begegnung mit den Dingen gerichtet sind, die mit uns unsere Welt konstituieren, gibt es in der Wahrnehmung Organisationstendenzen, die die Gegenstände auf uns hin gerichtet strukturieren, so dass die Vorzugsrichtungen, in denen statische Objekte wahrgenommen werden, nach links, nach oben und sagittal auf uns hin gerichtet sind.

$\mathrm{Da}$ wir uns als expansive Wesen in der horizontalen Ebene unterwegs befinden, unterschätzen wir die Geschwindigkeit eines Objektes, das sich in derselben Richtung wie unsere Orientierungsrichtung bewegt und überschätzen wir die Geschwindigkeit in entgegengesetzter Richtung. In der vertikalen Dimension befinden wir uns nicht unterwegs, sondern haben hier gleichsam den Endpunkt als Ruhepunkt erreicht, was unserem Dasein seine Stabilität garantiert. Daher findet keine Ưberschätzung der steigenden Bewegung statt. 